\title{
Identification of Caspase-6 as A Candidate Tumor Suppressor Gene at 4q25 in Oral Cancer
}

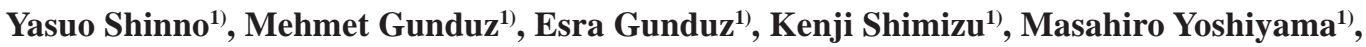 \\ Akira Yasuoka ${ }^{2)}$, Noriyuki Nagai ${ }^{1)}$ \\ 1)Okayama University, Graduate School of Medicine, Dentistry and Pharmaceutical Sciences \\ 2)Japan Institute for Advanced Dentistry, Osaka
}

\begin{abstract}
We recently reported the frequent deletion of chromosome 4q25 region in oral squamous cell carcinoma and discussed the Caspase- 6 as a possible tumor suppressor gene in this region due to its near location to this hot spot. We this time examined the role of Caspase-6 in oral cancers. mRNA expression level, protein localization and gene mutation of Caspase-6 were analyzed. Reverse-transcription-PCR analysis demonstrated decreased expression in $40 \%$ and equal expression in 30\% of Caspase- 6 mRNA of primary tumors as compared with that matched normal samples. cDNA sequencing analysis of Caspase- 6 did not reveal any somatic mutation. Caspase6 protein localization was in similar proportion with its mRNA expression. Failure of Caspase-6 response in tumor samples as compared to normal samples could suggest Caspase- 6 as a candidate tumor suppressor gene in a subset of oral cancer.
\end{abstract}

\section{Introduction}

In neoplastic progression, most of the sporadic solid tumors result from a multistep process of accumulated genetic and epigenetic alterations. Among these changes, inactivation of the tumor suppressor genes is one of the most critical steps. In this process, the deleted chromosomal regions eliminated one of the alleles, while inactivating events (mutation, deletion or promoter hypermethylation) affect the other allele of the concerning tumor suppressor genes ${ }^{1}$. Loss of heterozygosity analysis by using polymorphic microsatellite markers is a sensitive method to detect micro-deletions. We reported that loss of heterozygosity was found in high frequency in oral squamous cell carcinoma in a locus, which included Caspase- 6 gene. Caspase- 6 is in the most downstream of Caspase cascade, and in the apoptotic process, it is the gene, which carries out cell death by cutting off various substrates, for example nuclear membrane. It might be thought that aberration of apoptosis-related gene is concerned with cellular immortalization in carcinogenesis.

Therefore we this time examined the role of Caspase- 6 in oral cancers based on our previous study. We examined the mutation status and mRNA expression of Caspase-6 in oral squamous cell carcinoma samples. In addition, we examined p53 mutation status by immunohistochemistry and compared with caspase- 6 mRNA expression.

\section{Materials and methods}

Tissue Samples. Paired normal and tumor samples were obtained from 30 patients with primary oral squamous cell carcinoma, Okayama University Hospital after acquisition of informed consent from each patient.

RNA extractions. Total RNAs were prepared by using a modified acid guanidium phenol chloroform method (ISOGEN; Nippon Gene Co., Tokyo, Japan).

mRNA expression analysis. Thirty-two PCR cycles for Caspase-6 primers, RTS1 [5'-GGACACCAACATAACTGAGGTG] and RTAS1 [5'-AGCCGTTCACAGTTTCCCGGT] and 25cycles for GAPDH primers, S1 [5'-AGACCACAGTCCATGCCATCAC] and AS1 [5'-GGTCCACCACCCTGTTGCTGT], were used for amplification. We measured mRNA expression level of a Caspase-
6 gene using a computer based software (Quantity 1, Toyobo) semiquantitatively. The values of tumor specific Caspase-6 expressions were determined by calculating the ratio of the expression level in the tumor and that in the matched normal sample, each of which was normalized for the corresponding GAPDH expression level. Decreased and increased expression levels were defined when this ratio was $£ 0.75$ (25\% or more decrease) and ${ }^{3} 1.25$ (25\% or more increase), respectively. Reproducibility was confirmed by independent PCR repeated twice.

Mutation analysis. We analyzed p53, Capsase-6 gene by Direct Sequence after cDNA composition and did a mutation research. Immunohistochemistry. The tissue specimen fixed in 10\% neutral buffered formalin was submitted to Okayama University Hospital. Immunohistochemistry was performed on dewaxed paraffin sections using primary antibody of p53(NovoCastra, Newcastle, UK), Caspase-6(ABCAM, Cambridge, UK) and we used it and did DAB color development.

\section{Results}

Caspase-6 mRNA expression analysis showed normal level in $30 \%$ of cases, decrease of expression level in 30\%, and increase of expression level in 40\% (Fig.1). As a result of mutation analysis of Caspase-6, the mutation was not present.

Next, we studied localization of both protein of p53 and Caspase6 immunohistochemically. We studied mRNA expression analysis, mutation analysis and immunohistochemistry in 20 cases (fig.2). Caspase-6 protein localization was in similar proportion with its mRNA expression. In 7 of 20 cases, we found decrease of Caspase6 mRNA expression. In five of seven cases, p53 mutation was shown with an immunopositivity by immunohistochemistry. In cases without p53 mutation, caspase- 6 resembled positive in immunohistchemistry. In 5 of 20 cases, we found increase of Caspase- 6 mRNA expression. In three of five case, 53 mutation was not shown, and p53 was positive and caspase- 6 positive. In one case with p53 mutation both p53 and caspase- 6 were positive in immunohistochemistry.

\section{Discussion}




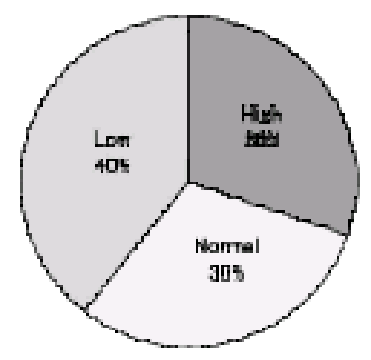

Caspase family genes were synthesized as procaspase-form. To become active, they were cleaved to two subunit, p20 and p10, by various activators ${ }^{3}$. Each Caspase family genes have specific activator. First, Caspase protein called Initiator Caspase receives a specific signal, and a Caspase cascade is activated. Various proteins, for example FAS, TNF, and p53, activate initiator Caspase. The active initiator Caspase activates familiar protein called effecter Caspase. The activated effector Caspase cuts various substrates that compose a cell, and apoptosis is completed. Caspase-6 is one of the effector Caspases. Therefore, we think that caspase- 6 did not have an important role in carcinogenic process. But, only Caspase-6, but not any other family genes, can cleave laminA of the composition to nuclear membrane ${ }^{4)}$. So, if Caspase-6 does not exist, nuclear membrane remains in the cell ever. These cells were not dead, but can't consider live.

In this study, $30 \%$ of the samples showed an increase of Caspase-6 mRNA expression in oral squamous cell carcinoma and $40 \%$ of cases had decreased expression. Caspase family gene expression may increase only at the time of apoptosis execution. Usually, the expression was very low, and protein is also in a precursor state ${ }^{5}$. We think that in remaining cases, Caspase-6 gene was mutated and generated unusual protein. Then, we examined mutation status of caspase- 6 by direct sequencing at the cDNA level. However, we cannot found any mutation in the coding region. Since we didn't perform sequencing at the genomic level, we may miss some of the mutant samples due to fragility of mutant mRNAs.

Most of the samples with decreased Caspase- 6 mRNA also demonstrated p53 protein positivity and caspase- 6 protein negativity by immunohistochemistry. In cases with increased Caspase- 6 mRNA expression, p53 gene was not mutated, and p53 protein, Caspase-6 protein were positive in the same location.

In oral squamous cell carcinoma, association was seen in

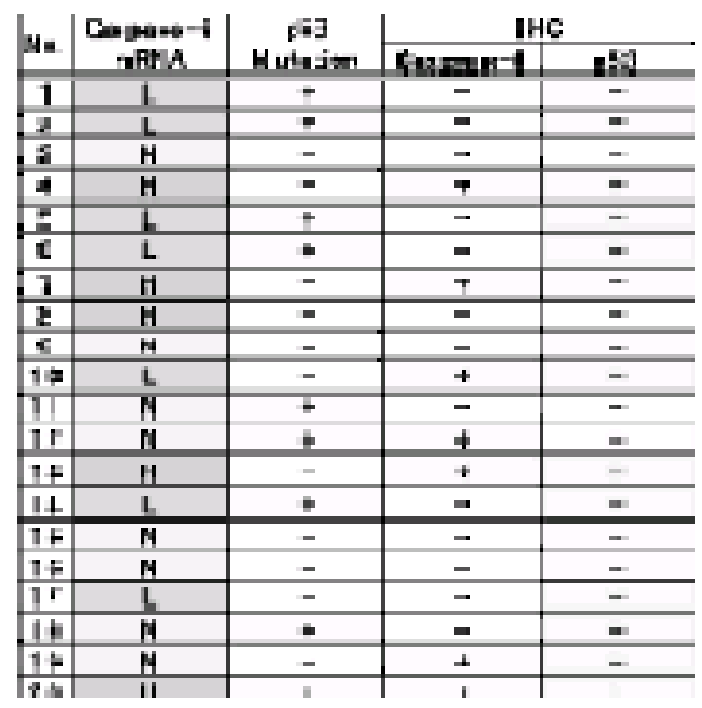

mutation of p53 with decreased expression of caspase-6. Caspase6 expression was also regulated by p53. Therefore, the reason for the negativity of caspase- 6 protein in p53 mutant cases could be due to loss of upstream signal by p53.

\section{References}

1) Knudson A.G.Jr.: Mutation and cancer: statistical study of retinoblastoma. Proc Natl Acad Sci U S A 68:820-823, 1971.

2) Fernandes-Alnemri T., Litwack G., Alnemri E.S.: Mch2, a new member of the apoptotic Ced-3/Ice cysteine protease gene family. Cancer Res 55:2737-2742, 1995.

3) Singh A.B., Kaushal V., Megyesi J.K., Shah S.V., Kaushal G.P. : Cloning and expression of rat caspase- 6 and its localization in renal ischemia/reperfusion injury. Kidney Int. 62 : 106-15, 2002. 4) Orth K., Chinnaiyan A.M., Garg M., Froelich C.J., Dixit V.M.: The CED-3/ICE-like protease Mch2 is activated during apoptosis and cleaves the death substrate lamin A. J. Biol. Chem. 271: 1644316446, 1996.

5) Omezzine A., Chater S., Mauduit C., Florin A., Tabone E., Chuzel F., Bars R., Benahmed M.: Long-term apoptotic cell death process with increased expression and activation of caspase- 3 and -6 in adult rat germ cells exposed in utero to flutamide. Endocrinology. 144 : 648-61, 2003. 